\section{Prevalence and management of canine Chiari-like malformation/ syringomyelia recorded in primary-care practice in England}

\section{Claire Thomas', Sandra Sandra Sanchis- Mora', Ludovic Pelligand', Holger Volk', David Church', Paul McGreevy², Peter Thomson' ${ }^{2}$, Dave Brodbelt' ${ }^{1}$, Dan O'Neill'}

The Royal Veterinary College, North Mymms, Hatfield, UK Faculty of Veterinary Science, The University of Sydney, NSW 2006, Australia

Chiari-like malformation/syringomyelia (CM/SM) describes a developmental disorder that can cause pain and reduce quality of life. This study aimed to explore clinical CM/SM cases in the veterinary primary-care setting in order to improve our understanding of the impact of the disorder on the overall canine population.

The study included clinical data on all dogs shared with the VetCompass primary-care project from 01/09/2009 to $13 / 06 / 2014$. The case definition required a clinical diagnosis with CM/SM confirmed using magnetic resonance imaging (MRI) in a clinically affected dog. Possible cases were sourced from the free text and VeNom diagnoses using key-search terms. Following manual verification, additional information was extracted regarding clinical signs and therapeutic management. Descriptive statistics were used to report CM/SM prevalence, demography, clinical signs and therapy.

A total of 59 CM/SM cases were identified from 187,365 dogs, giving a prevalence of $0.03 \%$ (95\% confidence interval (Cl): 0.02-0.04) across all dogs. The median (interquartile range) age at diagnosis was $4.33(0.33-11.58)$ years for the 34 incident cases $(57.62 \%)$. The most commonly affected breedtypes were Cavalier King Charles Spaniel (CKCS) (37 dogs; $62.71 \%$ ), King Charles Spaniels (12 dogs, 20.33\%), crossbreed (3 dogs, 5.08.\%), Yorkshire Terrier (2 dogs, 3.38\%), and Affenpinscher, Pug, English Toy Spaniel, Jack Russell Terrier and Chihuahua (1 dog, 1.69\%). From the VetCompass population of 3,860 CKCSs, this gave a breed prevalence of $0.96 \%$ (95\% Cl: $0.69-1.34)$ of MRI confirmed cases. Practitioners also reported an additional 84 CKCS $(2.18 \%, 95 \% \mathrm{CI}: 1.75-$ 2.70) suspected but unconfirmed cases.

The most common clinical signs associated with CM/SM were expression of pain (14 dogs, $23.72 \%$ ), characteristic CM/ SM-associated motor signs (scratching or facial rubbing without underlying skin disease) (11 dogs, 18.64\%) and ataxia or weakness (7 dogs, $11.86 \%$ ). Clinical signs were not specified for 16 cases $(27.11 \%)$

Analgesics (alone or in combination) were prescribed to 49 cases (83\%). The most common analgesic treatments were gabapentin (32 dogs, 65.3\%), NSAIDs (18 dogs, 36.7\%), glucocorticoids (30 dogs, $26.3 \%$ ) and tramadol (13 dogs, $26.5 \%$ ). Practitioners reported 31 dogs (63.26\%) showed apparent clinical improvement following treatment.

This study reported a low overall prevalence of clinical CM/ SM but higher prevalence in some individual breeds. The high proportion of affected animals with clinical signs that can variably reduce quality of life and that required analgesic therapy highlight the welfare impact of the disorder on affected dogs.

\section{Predisposing factors to proliferative ileitis in the Syrian hamster}

\section{Katie Charley', Erica Martin²}

Wolfgar Veterinary Surgery, Exeter, UK

Harper Adams University, Edgmond, UK

Proliferative ileitis in hamsters is associated with a grave prognosis, thus prevention is essential. The causative organism is Lawsonia intracellularis, which results in clinical disease during times of elevated stress. It is unknown whether L.intracellularis is carried by clinically healthy hamsters. There is limited scientific information on predisposing factors, including the important stressors. The aim of this prospective study was to identify if; weight, gender or number of hamsters housed within a $46 \times 28 \times 22 \mathrm{~cm}$ wire topped cage predispose hamsters to proliferative ileitis.

Data were collected from 475 hamsters arriving at a commercial pet supplier. A description of each was taken, this included markings, gender, weight and hamster groupings. Hamsters developing proliferative ileitis at the retailer or within one week of homing were diagnosed based on clinical signs of diarrhoea, dehydration and lethargy and identified by the researcher.
Gender had no significant influence on hamsters' predisposition to disease, therefore hamsters were considered irrespective of gender for following statistical analyses.

A statistically significant $(\mathrm{P}<0.001)$ correlation was discovered between increasing group size and incidence of disease where hamsters were in groups of two to six. No hamsters housed in groups of two $(n=18)$ or three $(n=75)$ developed disease, two percent (6) of hamsters in groups of four $(n=280)$, two percent (1) of hamsters in groups of five $(n=60)$ and $24 \%$ (10) of hamsters in groups of six $(n=42)$ developed disease.

Hamsters involved in this study weighed between $43 \mathrm{~g}$ and $120 \mathrm{~g}$. One-way ANOVA showed no significant difference $(P=0.822)$ in weights of hamsters contracting disease $(75.7 \mathrm{~g})$ compared to those not contracting disease (75.0g).

This study indicates that as group size rises, chance of contracting proliferative ileitis increases. Increased stress levels in hamsters housed within larger groups may be a causative factor. It is unclear from this study whether the limiting factor is number of cage mates or the space allowance per hamster; further research is necessary to identify this. Within the retail outlet weanling hamsters should be housed in groups no larger than three to reduce incidence of disease.

This study indicated that weight and gender are not predisposing factors to disease. Commercial pet suppliers can accept hamsters of both genders, weighing between $43 \mathrm{~g}$ and $120 \mathrm{~g}$ without fear of predisposition to proliferative ileitis. 